\title{
Accuracy of Self-Reported Arterial Hypertension in Brazil: Systematic Review and Meta-Analysis
}

\author{
Jessica Pronestino de Lima Moreira, ${ }^{1 \oplus}$ Renan Moritz Varnier Rodrigues de Almeida, ${ }^{2}$ Ronir Raggio Luiz ${ }^{10}$ \\ Instituto de Estudos de Saúde Coletiva, Universidade Federal do Rio de Janeiro - UFRJ,' Rio de Janeiro, RJ - Brazil \\ Instituto Alberto Luiz Coimbra de Pós-Graduação e Pesquisa em Engenharia, Universidade Federal do Rio de Janeiro - UFRJ, Rio de Janeiro, RJ - Brazil
}

\section{Abstract}

Background: Self-reported hypertension is a useful method to estimate prevalence in the population. However, it is necessary to evaluate its accuracy, in relation to the gold-standard diagnostic methods of the disease.

Objectives: To estimate combined measures of sensitivity and specificity for self-reported hypertension, using Brazilian validation studies that included gold standard methods.

Methods: A systematic review and a meta-analysis were developed. Two independent examiners evaluated 1389 and read 113 potentially eligible articles. Since self-reported morbidity is influenced by the cultural and economic characteristics of a population, as well as by its accessibility to medical care, only studies from one country (Brazil) were included. First, a qualitative analysis was performed, evaluating the relationship between self-reported hypertension and its measurement through gold-standard methods. Subsequently, a meta-analysis estimated the combined sensitivity and specificity for the included studies. Due to a high heterogeneity among studies, the metaanalysis used a random effects model. Bias risks were evaluated by the QUADAS-2 protocol and the standard significance level of $10 \%$ was used in all modelling.

Results: Five studies were included in the qualitative analysis; and four had the necessary information for inclusion in the meta-analysis. Patient selection and Index Test (the question allowing for self-reporting) were the domains with the highest risk of bias. In the meta-analysis, combined sensitivity and specificity were $77 \%(95 \% \mathrm{CI}:[74.5$ $79.0 \%])$ and $88 \%(95 \% \mathrm{CI}:[86.3-88.6 \%])$, respectively.

Conclusions: The analysed studies allowed for the estimation of more reliable values for combined sensitivity and specificity. These values were higher than those usually found in studies with greater population heterogeneity.

Keywords: Hypertension; Epidemiology; Meta-Analysis; Accuracy; Systematic Review.

\section{Introduction}

The estimation of systemic arterial hypertension $(\mathrm{SAH})$ through self-reporting questionnaires in populationbased surveys presents advantages, such as low cost, simplicity of measurement, and ease of application. However, as with any diagnostic test, classification errors are expected to occur, with inevitable false-positive and false-negative cases. Thus, a certain proportion of people will report having the disease when in fact they do not have it, and a group of people will report being healthy when they are actually sick.1,2

To quantify the accuracy of this procedure for SAH measurement, it is possible to calculate self-reported SAH sensitivity and specificity indicators, using a reference standard. The most common "gold standards" for this are the use of sphygmomanometers ${ }^{1,2}$ and of automatic measurement devices. ${ }^{3-5}$ Results found for sensitivity in these studies varied from $0.45^{4}$ to $0.84^{3}$, and, for specificity, from $0.81^{5}$ to $1^{4}$.

\section{Mailing Address: Jessica Pronestino de Lima Moreira}

Avenida Horácio Macedo, S/N - Próximo a Prefeitura Universitária da UFRJ. Postal Code: 21941-598, Ilha do Fundão - Cidade Universitária, Rio de Janeiro, 
Concerning the question used for self-assessment, most studies ${ }^{1-3}$ question if any doctor or health professional had ever claimed that the subject had arterial hypertension or "high pressure". As objective as this question may seem, there is always a degree of subjectivity within it, making its understanding difficult. For example, it is known that gender, age, schooling, the way the question is asked, and the type of interview (face-to-face or telephone) may influence answers, adding to the uncertainty introduced by the use of different gold standards and cut-off points for diagnosis.

In addition, the validation of self-reported morbidity is influenced by cultural and economic characteristics of a population, as well as by the operating health system. Self-reported morbidity depends on access to health care, and it may be assumed that populations with a similar health system (e.g. a "universal health system") will behave similarly in this respect.

In light of the above, the objective of this study was to estimate combined measures of sensitivity and specificity for self-reported SAH, using only studies performed in Brazil (therefore sharing similar cultural, economic, and access to health characteristics). A systematic review and a meta-analysis were developed for this purpose.

\section{Methods}

\section{Study Design}

This study is made up of systematic reviews and metaanalyses of diagnostic test accuracy, performed in Brazil, with the aim of estimating combined sensitivity (S) and specificity (E) from studies with validated self-reported hypertension.

\section{Eligibility criteria}

Participants: Studies that deal with arterial hypertension, with no restriction of age, gender, place of study, date, or language.

Intervention: Studies that used self-reference methods for SAH tracking, with no restriction as to the question or interview method (for instance, face-to-face or telephone).

Reference: Studies that diagnosed arterial hypertension by any method were considered to be the gold standard.

Outcome: Sensitivity and specificity of self-reported hypertension.

\section{Information sources}

The Cochrane Database of Systematic Reviews and other essential databases for primary health studies (WOS, Scopus, EMBASE, MEDLINE and LILACS) were searched until August 2020. Conference proceedings were also searched through the WOS, MEDLINE, and SCOPUS databases, and Theses/Dissertations from Brazil were searched in the CAPES Brazilian Theses and Dissertations Database. ${ }^{6}$

The search strategy included both controlled vocabulary and free terms (see appendix), and was first developed for MEDLINE via its PubMed interface, which was later adapted to the other databases. No language restriction was implemented, and researchers in the area (i.e. with published articles on the subject) were consulted via e-mail to find out if any study, to the best of their knowledge, had been missed by the search.

\section{Data Collection and Analysis}

Eligible studies were selected and duplicates were removed by reading the title and summary of the identified articles. Selected studies were then analyzed in their entirety, with the exclusion of those that did not have information necessary for the analysis or that did not use the defined cut-offs (systolic pressure $\geq$ $140 \mathrm{mmHg}$ and/or diastolic pressure $\geq 90 \mathrm{mmHg}$ ). Studies were also excluded when subjects were restricted to a sub-population, such as "women" "the elderly", "adolescents", or children".

Two independent reviewers assessed the studies and extracted the relevant data using a standardized form. In cases of incomplete data, attempts were made to contact the authors (e-mail). Cases of disagreement among reviewers were resolved by consensus after consultation with a third reviewer.

The self-reporting question used as an index test was: "Did any doctor or health professional ever say that you have hypertension or high blood pressure?". The evaluation of the risk of bias was done through the QUADAS-2 protocol, a Cochrane Collaboration tool for bias evaluation. ${ }^{7}$ Four domains of risk of bias were thus assessed: Patient Selection, Index Test, Reference Standards, and Flow and Time ${ }^{7}$. Regarding study implementation, three domains were evaluated: Patient selection, Index test, and Reference Standards. Each domain was classified as having a low, high, or unclear risk of bias. ${ }^{7}$ No need for blinding was deemed necessary, since self-reported SAH information would not influence SAH gold standard measurements. 
In addition, the following bias risk criteria were established:

- Non-random sample or sample based on participants with a pre-defined characteristic: high risk.

- Self-reporting question other than that defined above: unclear risk.

- No information on the reference standard used for SAH validation: unclear risk.

The included studies used the same cutoff point for disease definition (systolic pressure $\geq 140 \mathrm{mmHg}$ and diastolic pressure $\geq 90 \mathrm{mmHg}$ ); or indicated the use of antihypertensive medication. Authors whose works were eligible for the present study, but which did not have all the information necessary for the meta-analysis (number of true positives, false positives, true negatives and false negatives), were contacted by e-mail. Assessment of heterogeneity was performed by a visual inspection of forest plots, by the use of $\chi^{2}$ tests for heterogeneity, and by the Higgins Index $I^{2}$ (the proportion of true heterogeneity relative to the total variation of the estimated parameters). A random effects model was used in the presence of heterogeneity, defined as $\chi^{2}$, with a significance level < 0.10 and $\mathrm{I}^{2}>50 \% .^{8}$

All analyses were performed in the MetaDiSc software, v. 1.4 (Meta-analysis of Diagnostic and Screening Tests, Universidad Complutense, Madrid, Spain)..$^{9}$ It was defined that a meta-regression would be performed only if at least 10 eligible papers could be identified per covariate. ${ }^{10,11}$

\section{Results}

A total of 2,610 references were found through the database search. After the exclusion of duplicates, 1,388 studies were evaluated by title and abstract, and 113 studies were selected. These 113 studies were completely analyzed, and 24 were further selected. Of these, seven had been conducted in Brazil, one of these used only adolescent subjects and was excluded, and one of these used only elderly subjects and was excluded. Thus, five studies were finally included in the qualitative analysis,

Of the five studies above, four did not have all the necessary statistical information for a metaanalysis. E-mail contacts were successful in retrieving information for three of these, thus resulting in four studies for the meta-analysis.

Figure 1 shows the flow chart for the studies identified, screened, assessed for eligibility, excluded, and finally included in the analyses. No further study could be identified by personal (e-mail) consultation with researchers in the area.

Table 1 presents the characteristics of the studies in the systematic review and meta-analysis. The most common gold standards were sphygmomanometers. Table 2 shows the risk of bias in these studies. The domain with the smallest risk was "Flow and Time", and the domains "Patient Selection" and "Index Test" had the highest occurrence of high/unclear risk.

The forest plots for sensitivity and specificity according to the study are presented in Figure 2. The highest sensitivity was found in Chrestani et al. ${ }^{3}(\mathrm{~S}=84 \%)$ and the lowest in Louzada et al. ${ }^{4}(S=45 \%)$. By contrast, the latter presented a specificity of $100 \%$, with the lowest $\mathrm{E}$ found in Selem et al. $(\mathrm{E}=81 \%)$. The four studies that met the eligibility criteria are presented in Figures 2a and $2 \mathrm{~b}$. For both $\mathrm{S}$ and $\mathrm{E}$, a high degree of heterogeneity was found, with $\mathrm{I}^{2}>98 \%$ and a statistically significant $\chi^{2}$ ( $\left.\mathrm{p}<0.0001\right)$. Thus, a random effects model was used to estimate the combined value of $\mathrm{S}$ and $\mathrm{E}$, resulting in $\mathrm{S}=0.768$ (95\% CI: 0.745-0.790) and $\mathrm{E}=0.875$ (95\% CI: $0.863-0.886)$. The small number of studies hindered the development of a meta-regression to assess the influence of study characteristics on sensitivity/specificity or to evaluate publication bias.

\section{Discussion}

This study performed a systematic review of and a meta-analysis on self-reported $\mathrm{SAH}$ validation studies from Brazil. Five studies were included in the systematic review, four of which were eligible for the meta-analysis. The accuracy of the self-reported hypertension measurement can be considered satisfactory, as it was possible to correctly identify $77 \%$ of the people who truly had the disease (sensitivity), together with a specificity close to $88 \%$, showing a high capacity to detect true non-patients. This information, however, should serve as a warning to researchers dealing with population surveys, since it indicates that estimated self-reported hypertension may be quite divergent from real rates. The pooled sensitivity/ specificity results were high, and clearly superior to review studies conducted with more heterogeneous populations. For instance, one systematic review aiming to identify the proportion of knowledge of the disease (i.e. whether people with hypertension were aware of their condition) found a combined sensitivity 


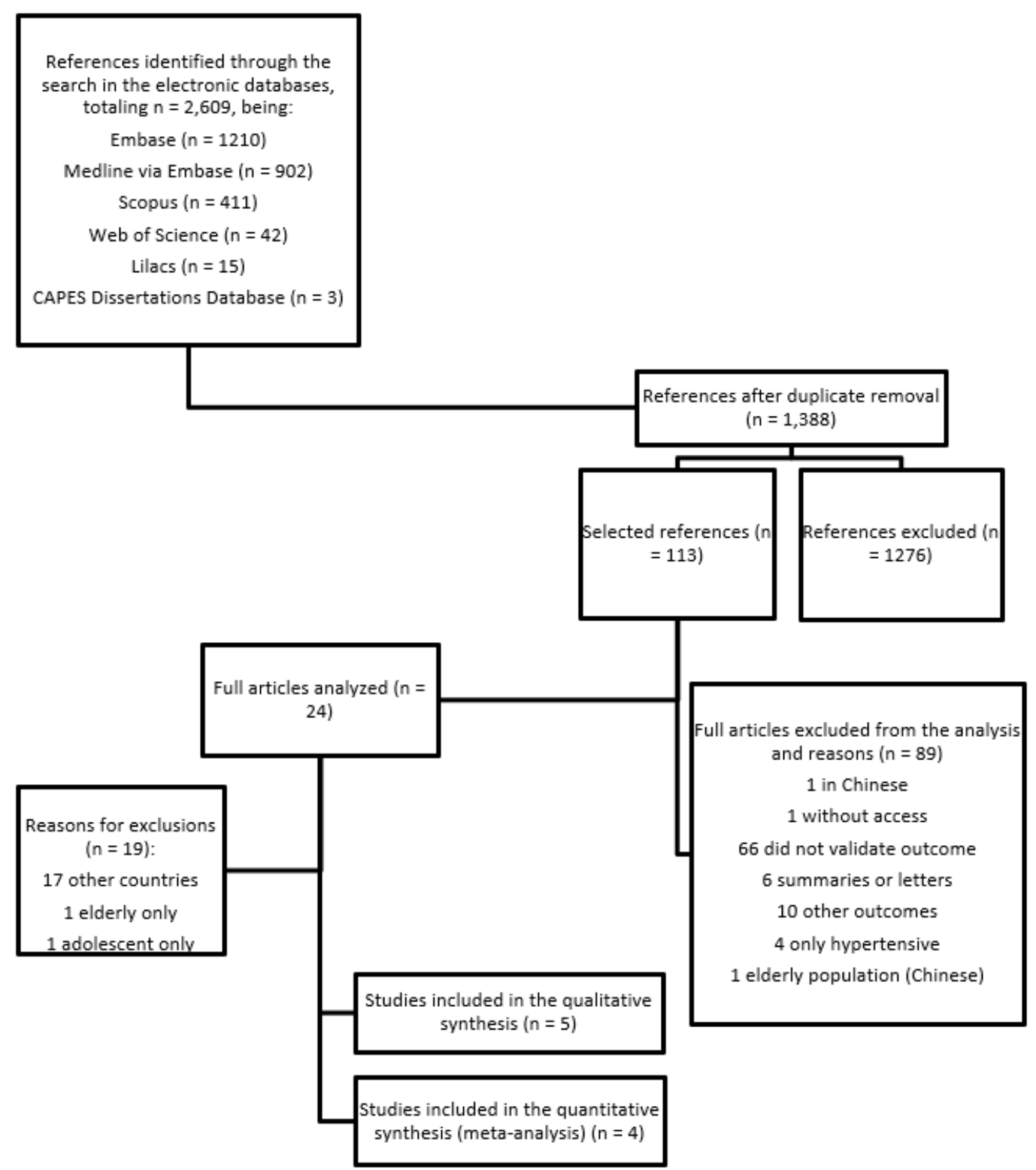

Figure 1 - Systematic review/meta-analysis study selection flow, self-reported SAH studies with reference standard validation.

Table 1 - Characteristics of the studies included in the systematic review and meta-analysis, self-reported SAH studies with reference standards

\begin{tabular}{lccccc}
\multicolumn{1}{c}{ Study } & $\begin{array}{c}\text { Use of defined } \\
\text { question }\end{array}$ & $\begin{array}{c}\text { Reference } \\
\text { standard }\end{array}$ & Samplesize & $\begin{array}{c}\text { Minimum } \\
\text { age }\end{array}$ & Sensitivity \\
\hline Lima-Costa et al, 2004 & Yes & Esfigmomanometer & 970 & 18 & 0.72 \\
\hline Chrestaniet al, 2007 & Yes & Authomatic monitor & 2906 & 20 & 20 \\
\hline Selem, et al 2013 & No & Authomatic monitor & 535 & 0.84 \\
\hline Louzada et al, 2010 & Not reported & Esfigmomanometer & 349 & No treported & 0.81 \\
\hline Campos et al, 2011 & Yes & Esfigmomanometer & 67 & 1.00 & 0.45 \\
\hline
\end{tabular}


Table 2 - Bias assessment through the QUADAS-2 protocol

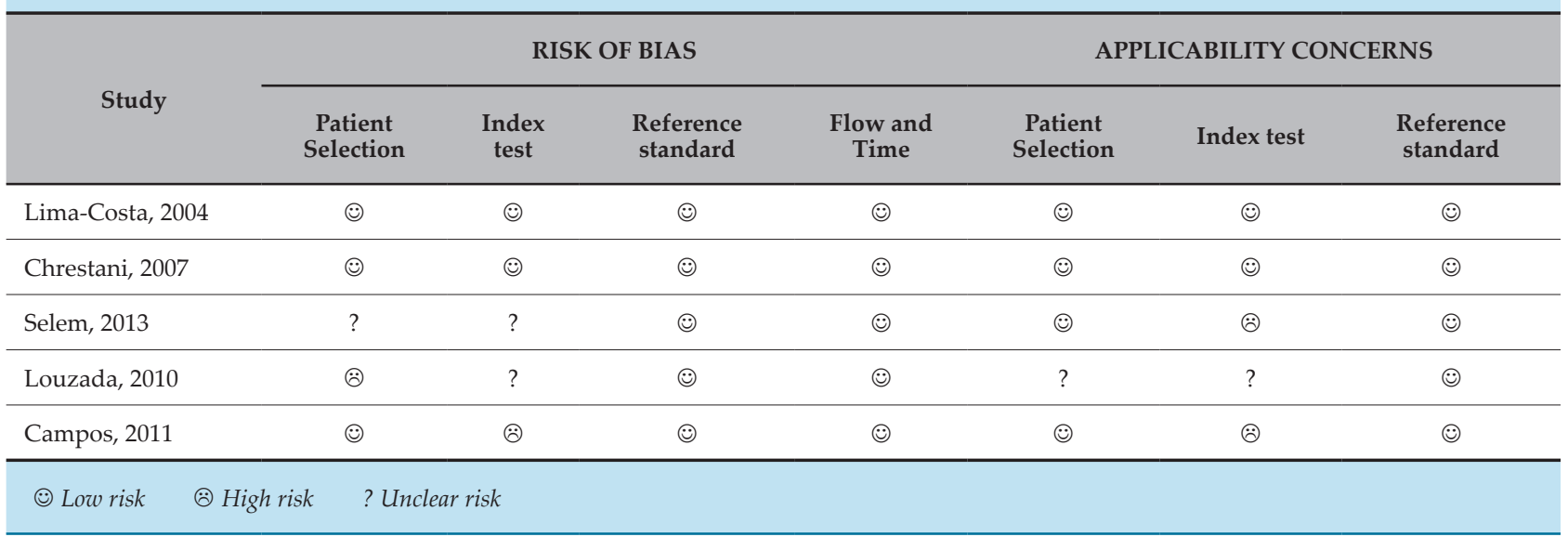

\section{SENSITIVITY}

2A. Brazil

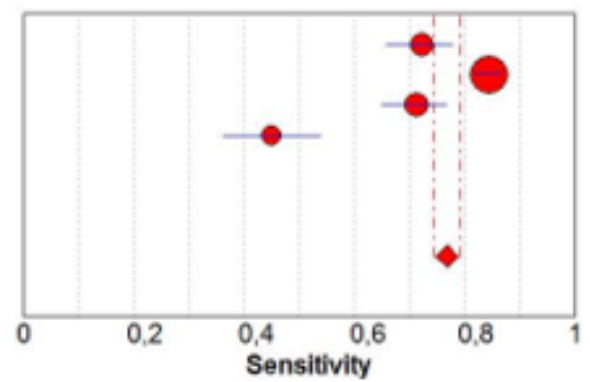

Polled sensitivity $=0.768(0.745-0.790)$

Chi-square $=96.04 ; g . l .=3(p<0.0001)$ Inconsistency $\left(1^{2}\right)=96.9 \%$

\section{SPECIFICITY}

\section{B. Brazil}
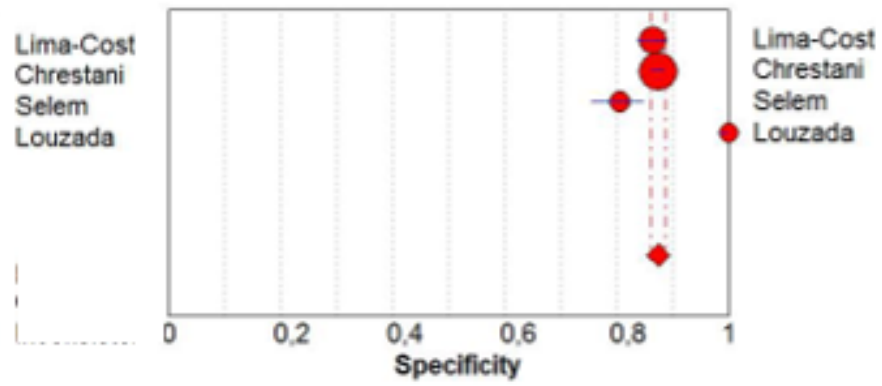

Polled specificity $=0.875(0.863-0.886)$

Chi-square $=71.83 ;$ g.I. $=3(p<0.0001)$

Inconsistency $\left(I^{2}\right)=95.8 \%$

Figure 2 - Forest plots of the self-reported hypertension validity studies using $\geq 140$ systolic pressure and/or $\geq 90$ diastolic pressure as the reference standard cut-off point, SAH diagnosis.

of $58.4 \%{ }^{12}$ but included studies from many countries. Another similar study used two of the papers analyzed here, finding a combined sensitivity of $42 \%$ and a specificity of $90 \%,{ }^{13}$ most likely due to the greater heterogeneity of its studies.

As expected, the study of a similar population (country) makes results more comparable, with a higher combined sensitivity. However, it should be noted that this problem does not arise in the validation of exam procedures, such as resonance versus tomography, since differences will mainly concern equipment validity and accuracy, and not the social and cultural characteristics of studied populations.

The use of self-reporting questionnaires to estimate the prevalence of hypertension may result in severe bias, and some studies present mathematical methods for dealing with this problem (e.g. ${ }^{14-16}$ ). However, knowledge on the sensitivity/specificity of measurement procedures in a specific population is a fundamental step in these correction methods. ${ }^{14-16}$ 
Therefore, the estimates presented here can be useful to obtain better HAS estimates in the analyzed population.

Brazil has a history of population-based health surveys, such as the Health Supplement of the National Household Survey (PNAD), which later gave rise to the National Health Survey (PNS); and VIGITEL - a telephone-based surveillance system for risk factors in chronic diseases, ${ }^{17}$ These studies have been conducted throughout the country, allowing, over the years, for a solid basis for epidemiological research and resulting in several self-reported morbidity studies.

Although the separate measures of sensitivity and specificity represented the main objective of this study, Lee et al. ${ }^{18}$ point out that analyzing these measures separately (disregarding their correlation) can produce incorrect results and that studies should use the same explicit diagnostic limit. ${ }^{18,19}$

A limitation of the present study was the small number of studies that fulfilled all the characteristics required for analysis. In addition, despite attempts of direct request, missing information for one of the eligible studies could not be recovered. Furthermore, reference tests, although considered as gold standards, may also have problems in the diagnosis of hypertension. In fact, the best method for SAH diagnosis (greater $S$ and E) is Ambulatory Blood Pressure Monitoring (ABPM). However, the complexity and time needed for ABPM application render its use difficult in population surveys, and apparently no study used this method as a gold standard for the validation of self-reported SAH.

\section{Conclusion}

Self-reporting has been frequently used for the population screening of $\mathrm{SAH}$, since it is considered to be a valid and relatively cheap procedure. This estimation method may result in bias, but this problem can be reduced with the help of the measurement sensitivity and specificity values. Therefore, the results presented here would allow for health policies based on more reliable data, as government agencies need to know the epidemiological profile of a population, and high-coverage surveys are commonly used for this purpose.

Although the combined values presented here were higher than those that would be used if only one of the analyzed studies were to be considered (sensitivity above $75 \%$ and specificity close to $90 \%$ ), researchers must take into account that the procedure still fails to detect a significant proportion of subjects with the disease.

In summary, the restriction to studies from a similar population allowed for a better characterization of the validity of self-reported hypertension, with combined sensitivity and specificity values higher than those found in studies conducted in several populations. Thus, systematic reviews of self-reported morbidity should consider the use of sub-groups in order to obtain more consistent estimates.

\section{Acknowledgements}

We are very grateful for the help of the librarian Danielle Masterson, from the Central Library of the Health Sciences Center of the Federal University of Rio de Janeiro, who was essential in the elaboration of the search strategies that gave rise to this study.

\section{Author contributions}

Conception and design of the research: Luiz RR, Moreira JPL, Almeida RMVR. Acquisition of data: Moreira JPL. Analysis and interpretation of the data: Moreira JPL, Almeida RMVR. Statistical analysis: Moreira JPL. Obtaining financing: Luiz RR. Writing of the manuscript: Luiz RR, Moreira JPL, Almeida RMVR. Critical revision of the manuscript for intellectual content: Luiz RR, Moreira JPL, Almeida RMVR.

\section{Potential Conflict of Interest}

No potential conflict of interest relevant to this article was reported.

\section{Sources of Funding}

This study was partially funded by Fundação Carlos Chagas Filho de Amparo à Pesquisa do Estado do Rio de Janeiro. Process n. E-26/102.357/2013 and E-26/224.838/2016.

\section{Study Association}

This study is not associated with any thesis or dissertation work.

\section{Ethics approval and consent to participate}

This article does not contain any studies with human participants or animals performed by any of the authors. 


\section{References}

1. Campos, SF. Validity and reproducibility of anthropometric measurements, reported morbidity, consumption of food and beverages obtained by telephone survey. Minas Gerais: UFMG; 2011.

2. Lima-Costa MF, Peixoto S V, Firmo JOA.Validity of self-reported hypertension and its determinants (the Bambuí study). Rev Saude Publica, 2004;38(5):637-42.

3. Chrestani MAD, Santos IS, Matijasevich AM. Self-reported hypertension: Validation in a representative cross-sectional survey. Rep Public Health, 2009; 25(11):2395-406.

4. Louzada JCA,Andrade RM, Dionísio EJ, Barel M, Monteiro HL, Amaral SL. Comparison between self-reported hypertension and casual blood pressure and presence of cardiovascular risk factors among health workers of Bauru and Jaú cities. Medicina, 2010;43(4):408-18.

5. Selem SSC, Castro MA, César CLG, Marchion DML, Fisberg RM. Validity of self-reported hypertension is inversely associated with the level of education in Brazilian individuals. ABC Cardiol J., 2013;100(1):52-9.

6. Brasil.Ministério da Saúde. Methodological guidelines: elaboration of a systematic review and meta-analysis of studies of diagnostic accuracy. Brasília: 116 .

7. Whiting PF, Rutjes AWS, Westwood ME, MallettS, Deeks JJ, Reitsma JB, et al.QUADAS-2: A Revised Tool for the Quality Assessment of Diagnostic Accuracy Studies. Ann Intern Med. 2011;155(8):529-36.

8. Pereira MG, Galvão TF. Heterogeneity and publication bias in systematic reviews. Epidemiol Health Serv. 2014;23(4):775-8.

9. Zamora J, Abraira V, Muriel A, KKhalid, Coomarasamy A. Meta-DiSc: a software for meta-analysis of test accuracy data, BMC Med Res Meth. 2006; 6:31
10. DeVet, HCW et al. Cochrane Handbook for Systematic Reviews of Diagnostic Test Accuracy. [s.1.] The Cochrane Collaboration, 2008.

11. Higgins JPT, Green S. Cochrane Handbook for Systematic Reviews of Interventions I Cochrane Trainin,; 2011.

12. Gorber SC, Tremblay M, Campbell N, Hardt J. The accuracy of selfreported hypertension: A systematic review and meta-analysis. Curr Hypertens Rev. 2008;4(1):36-62.

13. Gonçalves VSS, Andrade KRC, Carvalho KMB, Silva MT, Pereira MG, Galvao TF. Accuracy of self-reported hypertension. Hypertens, 2018;36(5):970-8.

14. Lew RA, Levy PS. Estimation of prevalence on the basis of screening tests. Statist Med. 1989;8(10):1225-30.

15. Moreira JPL,Almeida RMVR, Rocha NCS, Luiz RR. Correction of selfreported prevalence in epidemiological studies with large samples. Rep Publ Health, 2016;32(12):21

16. Rogan WJ, Gladen B. Estimating prevalence from the results of a screening test. American Journal of Epidemiology, 1978, 107(1);71-6.

17. Malta DC, Leal MC, Costa MFL, Morais Neto OL. National Health Surveys: accumulated experience and proposal for the Brazilian health survey. Brazilian J Epidemiol. 2008;11(suppl 1):159-67.

18. Lee J, Kim KW, Choi SH, Huh J, Park SH. Systematic Review and MetaAnalysis of Studies Evaluating Diagnostic Test Accuracy: A Practical Review for Clinical Researchers-Part II. Statistical Methods of MetaAnalysis. Korean J Radiol.2015;16(6):1188-96.

19. Egger M, Smith GD, Altman DG. Systematic reviews in health care: meta-analysis in context. London: BMJ Books;2007. 